Pinard M-H, Gay C, Pastoret P-P, Dodet B (eds): Animal Genomics for Animal Health. Dev Biol

(Basel). Basel, Karger, 2008, vol 132, pp 425-427.

\title{
Key Recommendations
}

\section{Quantitative Genetic Studies to Identify Markers of Health TRAITS}

Problem areas:

- Availability and acquisition of appropriate and reliable health records;

- $\quad$ Availability and acquisition of animal pedigrees with defined phenotypes;

- Development and availability of genome associated research tools; and

- $\quad$ Refining quantitative population genetic studies to usable markers.

\section{Recommendations:}

1. Support research to define health traits at the molecular level with the aim of detecting genetic factors involved in disease susceptibility and disease resistance.

2. Support initiatives at every level (national, regional, private, and research project) that aim to proactively record defined health traits and collect samples from either commercial populations, well-characterized experimental animal lines, or where livestock and poultry are exposed to environmental or epizootic extremes.

3. Support the development of inter-operable databases for large-scale storage of pedigree information, phenotypes, and associated environmental factors.

4. Support national and international efforts that aim to sequence animal genomes, develop genome-enabled technologies, and enable their cost-effective use in research.

5. Support the building and integration of bioinformatics infrastructures in animal health research institutions, including the development of statistical models and software for animal genomics research.

6. Support research programmes that focus on the the selection of animals with superior resistance to the immunosuppressive effects of stress.

7. Support large-scale coordinated research programmes aimed at elucidating relevant aspects of host-pathogen interactions for high priority diseases.

8. Support meetings and workshops to enhance communication and facilitate collaborations between quantitative geneticists and experts in animal health.

\section{Functional Genomics to Understand Host-Pathogen InTERActions}

\section{Problem areas:}

- Availability of validated animal disease models;

- Animals with defined disease susceptible/disease resistant phenotypes; 
- Bioinformatics tools;

- Identification of genes that are involved in the activation/repression of key regulatory pathways;

- Development and availability of reagents to study gene function; and

- Discovery of biomarkers.

\section{Recommendations:}

1. Support the development of experimental animal challenge models that provide measurable phenotypes relevant to actual diseases under field conditions.

2. Support long-term projects that will build the infrastructure needed to conduct the extensive phenotyping and genotyping of animal populations relevant to animal health priorities.

3. Support research programmes that aim to identify genetic markers that correlate with disease phenotypes, and carry out whole genome expression analyses using tissue-specific microarrays to identify genome sequences that influence disease parameters.

4. Support functional genomics research programmes that aim to identify genetic markers that are linked to regulatory pathways that modulate host-pathogen interactions.

5. Support research programmes in relevant animal hosts that aim to confirm the association of genetic markers with host responses in large animal populations.

6. Support the establishment of core bioinformatics infrastructures with state-ofthe-art genomic tools and resources that can integrate genotype, phenotype and functional genomics data for agricultural animal species and their respective priority diseases.

7. Support genome database initiatives for priority animal species and pathogens.

8. Develop proteomics tools to support animal health research projects that have the aim of identifying host molecules and biomarkers that are associated with or have a role in modulating host-pathogen interactions.

9. Establish an international consortium to advance and enable the development of reagents to study gene regulation, gene function, and disease pathways in priority animal species.

10. Support initiatives to integrate genetic, functional genomic, and bioinformatics data in a systems biology approach to understand complex diseases.

\section{Translating Genomic Information to ToOls for Controlling DISEASES}

\section{Problem areas:}

- Using animal genomes to understand genetic diversity and inform best management practices to improve animal health and animal production systems worldwide;

- Easy to use diagnostic platforms for genetic tests, forensic veterinary medicine, and traceability;

- Selection of animals with disease susceptible and resistant traits; and

- Application of genome-enabled technologies in drug and vaccine discovery, including transgenic animals that are resistant to disease and the selection of animals that respond effectively to drugs and vaccines. 


\section{Recommendations:}

1. Support initiatives that will use animal genomic innovations to assess and manage domestic and wildlife animal genetic diversity.

2. Support studies that will lead to the development of rapid, accurate and costeffective molecular genetic tests for host and pathogens to analyze field disease outbreaks and to develop predictive diagnostics.

3. Integrate disease resistance traits with existing multi-trait selection programmes.

4. Integrate genetic profiling in the development of disease models for drug and vaccine discovery research.

5. Support initiatives that integrate genomic approaches to vaccine development, including genetic and immune variations in target animal populations.

6. Support transgenic animal research programmes that target disease resistance.

7. Support research programmes that include collaborations between animal health research institutions, commercial breeding companies, and pharmaceutical companies.

\section{InTEgrating Stakeholder Support to Advance Animal Genomics in ANIMAL Health}

\section{Problem areas:}

- Collaborations between the public and private sectors;

- Intellectual property and technology transfer to industry;

- Impact on veterinary education and animal and poultry science;

- Contributions of veterinary practitioners, animal health professionals, and animal agriculture allied industries;

- Support from regulatory agencies to enable these new technologies; and

- A lack of funding targeting the application of genomics in animal health research.

\section{Recommendations:}

1. Support the funding of innovations in animal genomics research specifically designed to solve problems in animal health.

2. Develop integrated approaches that include the development of sample repositories and databases that support research networks.

3. Develop more "epidemiological" approaches to the genetics of animal health and integrate them into veterinary and animal science educational programmes.

4. Establish and support demonstration projects to deliver concrete examples of the value of integrating genome-enabled technologies in animal health research programmes.

5. Establish a progressive international regulatory framework that is science-based and provides a clear path for registering animal disease control tools derived from genome-enabled technologies.

6. Support meetings to align veterinarians, animal scientists, and genomics/genetics experts to foster a dialogue and educate on the importance of multidisciplinary approaches addressing the application of genome-enabled technologies to animal health. 